\title{
CHIRPED FIBER BRAGG GRATING AS ELECTRICALLY TUNABLE TRUE TIME DELAY LINE
}

\author{
Vincenzo Italia ${ }^{1}$, Marco Pisco ${ }^{1}$, Stefania Campopiano ${ }^{1}$, Andrea Cusano ${ }^{1}$ and \\ Antonello Cutolo ${ }^{1}$ \\ ${ }^{1}$ Divisione di Optoelettronica - Dipartimento di Ingegneria, Università del Sannio, \\ Corso Garibaldi 107 Benevento, Italye-mail stefania.campopiano@unisannio.it
}

\begin{abstract}
A new optical time delay line based on a Chirped Fiber Bragg Grating is proposed. Numerical results show the time delay can be electronically varied by changing the grating temperature with a minimum step of $1 \mathrm{ps}$ up to $30 \mathrm{GHz}$.
\end{abstract}

\section{INTRODUCTION}

During the last years, optical true-time delay (OTTD) units have been investigated for wideband squint-free beamforming for phased array antennas [1][3]. The advantages of the optical beamforming networks are quite well know: low insertion loss, high phase stability, electromagnetic interference immunity and low mass and volume.

Recently, fiber Bragg gratings (FBG) have been used to realize OTTD units. The first approaches were based on a number of uniform FBG written at different positions on optical fibers and the distances between gratings determine the beampointing direction of the array antenna [4]. This system assures broad-band operation, but it only allows a discrete number of beampointing angles.

Subsequently, it has been demonstrated that linearly chirped fiber gratings (LCFG) can produce a linear phase delay of the modulating signal at microwave frequencies and the slope of the phase response can be continuously modified by 
tuning the wavelength of the optical carrier [5]. Broadband operation and continuous spatial scanning properties have been demonstrated in these systems. However these systems are very complicated as the need to use multiwavelength tunable laser source and tunable bandpass filter that should be tuned synchronously with wavelengths of the tunable laser source.

In this work, we propose and numerically analyze a novel variable OTTD based on a LCFG operating at a fixed optical wavelength that simplifies the architecture of the beamforming system. The operation principle, based on uniform temperature perturbation of a single LCFG, is illustrated in Section 2. As consequence of the temperature perturbation, the grating complex amplitude reflectivity moves rigidly, causing a delay's variation for the input optical carrier. The different time delays are obtained by changing the grating temperature. In Section 3 the numerical results of the proposed OTTD are presented.

\section{PRINCIPLE OF OPERATION}

The proposed OTTD is illustrated in Figure 1. It's based on the use of one single LCFG uniformly perturbed in temperature.

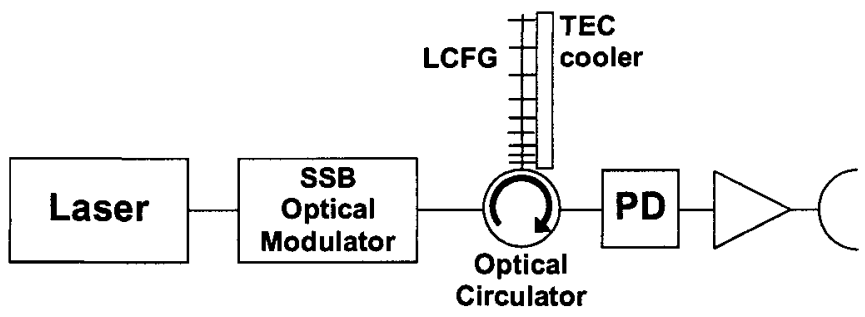

Figure 1. Single branch, OTTD based on a LCFG uniformly perturbed.

When a grating is uniformly perturbed in temperature its complex amplitude reflectivity is moved rigidly and the new Bragg wavelength is [8],

$$
\lambda_{B}(T)=\lambda_{B_{0}}+k_{T} \lambda_{B_{0}}\left(T-T_{0}\right),
$$

where $T_{0}$ is the room temperature, $\left(T-T_{0}\right)$ is the variation of the temperature introduced by the actuator, $\lambda_{B_{0}}$ is the unperturbed Bragg wavelength and $k_{T}$ is a constant determined by the characteristics of the grating. Figure 2 shows the 
temperature induced reflectivity and group delay shift as a function of the optical wavelength.
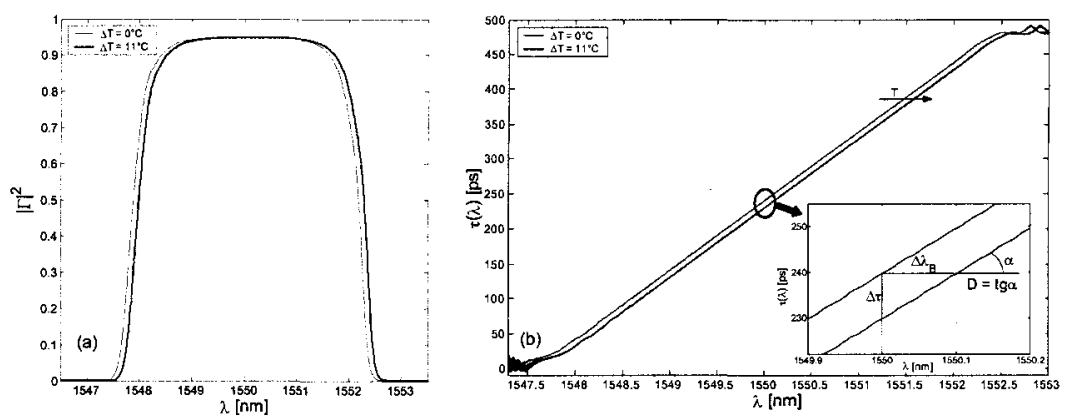

Figure 2. Reflectivity (a) and Time delay (b) responce for two values of temperature variation $\Delta T\left(\Delta T=0^{\circ} \mathrm{C}, \Delta T=11^{\circ} \mathrm{C}\right)$

Assuming the wavelength of the optical carrier equals to the unperturbed Bragg wavelength, $\lambda_{0}=\lambda_{B_{0}}$, the actual time delay as a function of the thermal changes, as shown in Figure 2, can be expressed as:

$$
\tau\left(\lambda_{B}\right)=\left.\tau\left(\lambda_{B}\right)\right|_{\Delta T=0}-D \cdot \Delta \lambda_{B}=\left.\tau\left(\lambda_{B}\right)\right|_{\Delta T=0}-k_{T} \lambda_{B} D \cdot \Delta T
$$

where $D$ is the mean FBG group-delay slope and $\Delta \lambda_{B}$ is the Bragg wavelength shift due to the temperature variation. If the carrier is amplitude modulated by a microwave signal of frequency $f_{R F}$, the modulated signal suffers a phase delay given by [3]

$$
\Delta \Phi_{R F}=2 \pi f_{R F} \tau\left(\lambda_{0}, T\right)
$$

Hence, the grating produces a linear phase shift in the modulating signal and the phase slope can be continuously varied by changing the temperature.

The drawbacks in the use of the LCFG as a wide bandwidth OTTD unit can be identified in the spectral distortion and in the RF power degradation due to chromatic dispersion induced by the grating phase response. The first problem can be neglected in practical applications due to limited bandwidth of the RF modulation. For the second one optical losses can be significant if DSB amplitude modulation is used. As illustrated in detail in the following section, this problem can be solved by using SSB modulation [3]. 


\section{NUMERICAL RESULTS}

In the modelling of the device, we used a 5-cm-long chirped grating with its bandpass centered at $\lambda_{\mathrm{B} 0}=1550 \mathrm{~nm}$ and a $3-\mathrm{dB}$ bandwidth of $4.32 \mathrm{~nm}$. The mean group-delay slope is $100 \mathrm{ps} / \mathrm{nm}$ with a mean group delay ripple (GDR) of $0.1 \mathrm{ps}$. The reflectivity and the time delay response of the grating are shown in Figure 3.

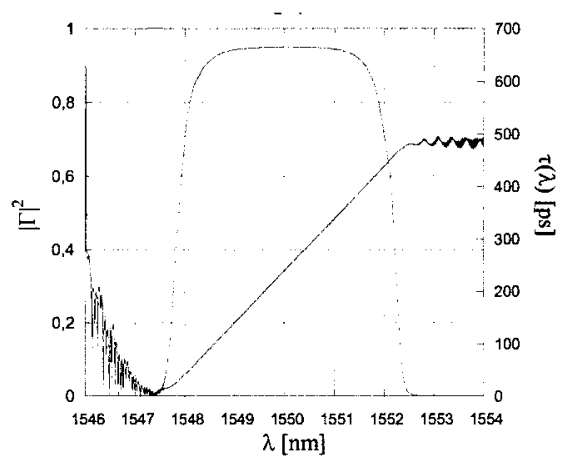

Figure 3. Grating reflectivity and time delay response of $L C F G$.

To obtain this GDR value an apodized LCFG with a positive hyperbolic-tangent apodization profile [6] has been used. An iterative method of GDR correction [7] has been used too. The apodization enabled a first and drastic reduction of the GDR while the iterative technique has been used to reduce the mean amplitude of the ripples under $0.1 \mathrm{ps}$ value. For thermal sensitivity of LCFG a value of $5.76 \times 10^{-6}$ [9] is chosen for $k_{r}$. Grating complex reflectivity has been evaluated by using multilayer approach [10].

Delay line's simulations have been performed for temperature varying from room temperature $\mathrm{T}_{0}$ to $\left(\mathrm{T}_{0}+11^{\circ} \mathrm{C}\right)$ with steps of $1.1^{\circ} \mathrm{C}$ to introduce variable delays from $1 \mathrm{ps}$ to $10 \mathrm{ps}$ with a step resolution of $1 \mathrm{ps}$ and SSB optical modulation have been used. Figure 4 shows the Bragg wavelength shift of the grating as the function of temperature perturbation. It presents a linear dependence on perturbation according to (1). 


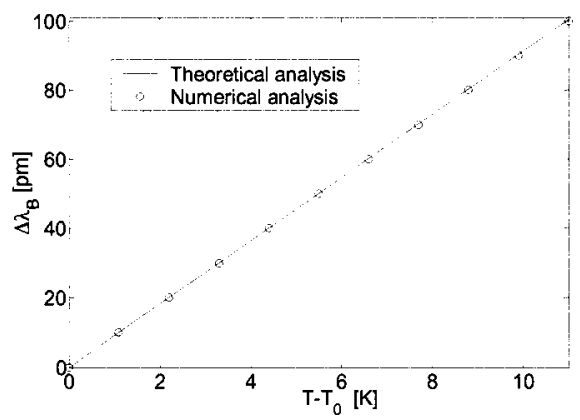

Figure 4. Bragg wavelength shift as a function of temperature perturbation.

In order to show the potential broad-bandwidth of the system the numerical simulations were made at different microwave frequencies in the range [1-30 $\mathrm{GHz}]$.

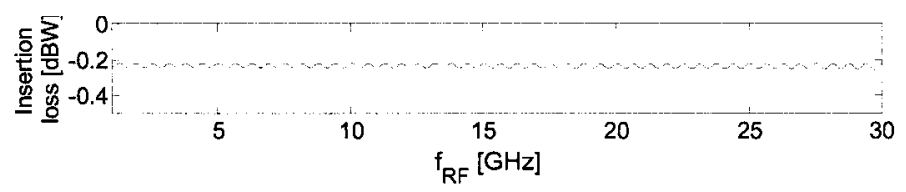

Figure 5. Insertion loss of the proposed OTTD as a function of detected frequency $\left(f_{R F}\right)$

Figure 5 shows the insertion loss of the OTTD at different modulating frequencies obtained only considering the losses of the grating and neglecting the contributions of the other optical devices in the chain. The mean value of the evaluated insertion losses is due to the limited reflectivity of the grating.

In order to verify the time-delay behaviour described in eq. (2), the phase of the detected signal has been estimated as a function of RF frequency in the $1-30 \mathrm{GHz}$ range for all values of perturbation. 


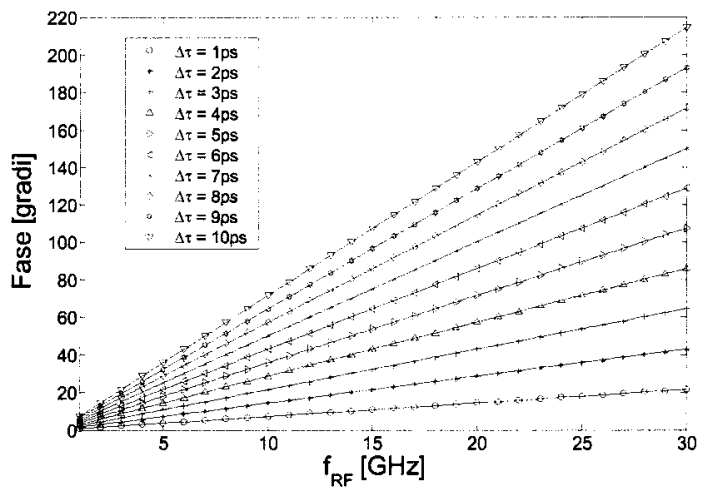

Figure 6. - Phase of the microwave signal at different $R F$ frequencies after reflection by the LCFG for all values of perturbation.

Figure 6 shows the relative detected phase of the microwave signal after reflection in the grating. It presents a linear dependence on the frequency, with different slopes according to eq. (2).

In order to check the time-delay independence from detected frequency, the ripple around the ideally constant group delay has been calculated. The result is plotted in Figure 7 showing a worst-case standard deviation of 0.07 ps from 1 to $30 \mathrm{GHz}$.

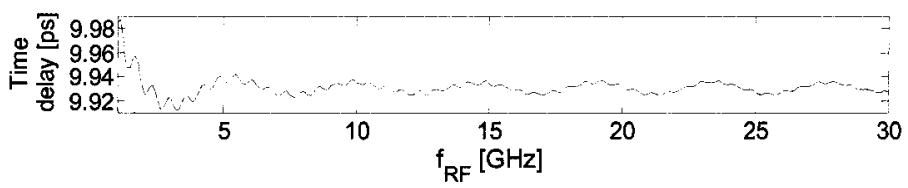

Figure 7. Time-delay ripple at against detected frequency (fRF) for temperature perturbation of $10^{\circ} \mathrm{C}$.

The total system time-delay error should be estimated assuming two main causes of error: the temperature stability $\Delta T_{a t t}$ and the FBG time-delay response deviation from the linear slope $\sigma_{\text {ripple }}$. The temperature stability can be translated to time precision by taking into account the grating time delay response

$$
\sigma_{\text {att. }}=k_{T} \lambda_{B} D \cdot \Delta T_{\text {att }}
$$

A standard deviation of $0.07 \mathrm{ps}$ would resume the time delay uncertainty due to the grating group-delay ripple. Taking into account both effects (temperature stability and the FBG group delay response) a total system time-delay precision, 
for assuming a temperature stability of $0.1^{\circ} \mathrm{C}$, is $\sim 0.1 \mathrm{ps}$, which in turn implies a time-delay equivalent to a $3^{\circ}$ phase error at $30 \mathrm{GHz}$.

\section{CONCLUSIONS}

In this work, we have presented a new chirped fiber grating beamformer. The device operates at a fixed optical wavelength and the time delay is linearly tuned by simply changing the grating temperature.

Numerical results show that the time delays is linearly proportional to the temperature, according to the theoretical analysis. The proposed system can be used for wideband beamforming at radio frequencies up to $30 \mathrm{GHz}$ with a 1-ps minimum time delay.

Inaccuracies due to the temperature stability and the linearity of the chirped grating group delay have also been considered, and their impact on the PAA performance is reported.

\section{REFERENCES}

[1] Istvàn Frigyes, Senior Member, IEEE, and A.J. Seeds, Senior Member, IEEE, "Optical Generated True-Time Delay in Phased-Array Antennas," IEEE Trans. on Microwave Theory and Techniques, vol. 43, no. 9, September 1995.

[2] J.L. Corral, J. Martì, Member, IEEE, S. Regidor, J.M. Fuster, R. Laming, M. J. Cole, "Continuously Variable True Time-Delay Optical Feeder for Phased-Array Antenna Employing Chirped Fiber Gratings," IEEE Tran. on Microwave Theory and Techniques, vol. 45, no. 8, August 1997.

[3] B. Ortega, J.L. Cruz, J. Capmany, M.V. Andrés, and Daniel Pastor, "Analysis of a Microwave Time Delay Based on a Perturbed Uniform Fiber Bragg Grating Operating at Constant Wavelength," J. of Lightwave Technology, vol. 18, no. 3, March 2000.

[4] A. Molony, C. Edge, and I. Bennion, "Fiber grating time delay for phased array antennas," Electron. Lett., vol. 31, pp. 1485-1486, 1995.

[5] J. L. Cruz, B. Ortega, M.V. Andrés, B. Gimeno, D. Pastor, J. Capmany and L. Dong, "Chirped Fibre Bragg Gratings fr Phased Array Antennas," Electron. Lett., vol. 33, no. 7, March 1997.

[6] K. Ennser, M. N. Zervas, and R.I. Laming, "Optimization of Apodized Linearly Chirped Fiber Gratings for Optical Communications," IEEE J. of Quantum Elettron. vol. 34, no. 5, May 1998.

[7] M. Sumetsky, P.I. Reyes, P.S. Westbrook, N.M. Litchinitser, and B.J. Eggleton, "Group Delay Ripple Correction in Chirped Fiber Bragg Gratings," Opt. Lett., vol. 28, no. 10, May 2003.

[8] V. Bhatia, "Properties and Sensing Applications of Long-Period Gratings", Ph.D. Thesis, November 1996.

[9] N.P. Bansal et al., Handbook of Glass Properties, Academic Press, Florida, 1986.

[10] Raman Kashyap Fiber Bragg Gratings, Academic Press, 1999. 